\title{
Aplicación de la capacidad bactericida del cobre en la práctica médica
}

\author{
VALERIA PRADO J. ${ }^{1}$, ROBERTO VIDAL A. ${ }^{1, a}$, CLAUDIA DURÁN T.1,
}

\section{Application of copper bactericidal properties in medical practice}

Copper is essential for cell metabolism in animals and plants and thus for life. Along centuries, copper has been identified as a metal containing antimicrobial properties. In recent years, laboratory assays and clinical studies have revealed that surfaces of metallic copper or its alloys, containing at least 70\% copper, eliminate in a few hours several pathogenic organisms including bacterial strains associated with nosocomial infections, influenza virus, HIV, and fungi such as Candida albicans. In March 2008, the American Environmental Protection Agency (EPA), supported by scientific evidence gathered to date, registered copper as the first and only metal with antimicrobial properties. We herein review certain mechanisms proposed for the antibacterial, antiviral and antifungal activity of copper. We also discuss in vitro and clinical efficacy studies developed world wide and in Chile, focusing on bactericidal activity of copper surface areas in comparison to materials typically used in hospital environments such as stainless steel and polymers. Scientific evidence gathered to date, consistently shows that the use of copper surface areas in high contact critical points in hospitals, significantly reduces environmental bacterial load. This is associated with a decreased risk of pathogen transmission to patients and represents therefore an interesting complement to infection control programs.

(Rev Med Chile 2012; 140: 1325-1332).

Key words: Anti-infective agents; Chile; Outpatient clinics, hospital.

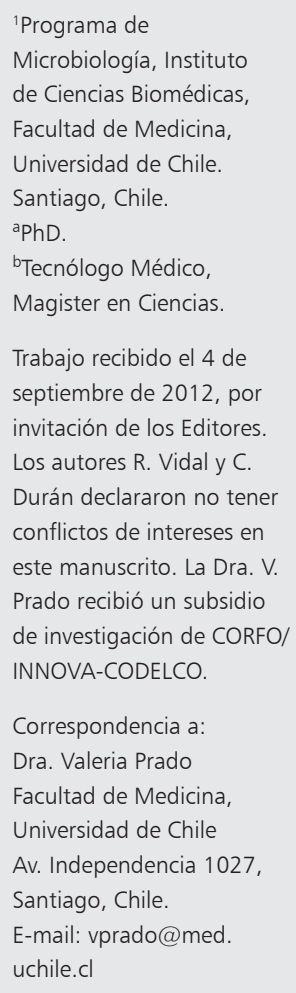

E l cobre ha acompañado a la humanidad desde sus inicios, ya que es uno de los metales que, en bajas concentraciones, son esenciales para el metabolismo de las células animales y vegetales.

Sus propiedades para prevenir infecciones eran reconocidas desde la antigüedad. A comienzos del siglo $\mathrm{XX}$, previo a la era antibiótica, diferentes compuestos químicos de cobre fueron utilizados en el tratamiento de patologías infecciosas, como impétigo, tuberculosis y sífilis ${ }^{1}$.

En la última década se ha revitalizado el concepto del cobre como antimicrobiano, se han realizado estudios de laboratorio y a nivel clínico para demostrar su eficacia, por lo cual en los próximos años se espera que el cobre se integre a la infraestructura hospitalaria como un elemento de ayuda en la lucha contra las infecciones. Para Chile, principal productor de cobre en el mundo, es un tema de alto interés.

En este artículo se revisarán las evidencias disponibles.

\section{Presencia del cobre en la actividad humana}

El cobre, junto con el oro, fueron los primeros metales utilizados por el hombre, actividad que se inició hace más de 9.000 años. En la actualidad el consumo de cobre a nivel mundial es del orden de 18 millones de toneladas anuales $(2,6 \mathrm{~kg}$ per cápita, estimando una población mundial de 6.800 millones). Este uso solamente es superado por el hierro y el aluminio (WBMS, 2008, Oficina Mundial de estadística del metal). 
En la vida cotidiana son conocidas y apreciadas algunas características del cobre que facilitan el funcionamiento de las sociedades modernas, entre ellas su capacidad de ser un buen conductor de la electricidad y buen conductor térmico; por ello el cobre tiene un amplio uso en áreas industriales relacionadas con fabricación de automóviles, climatización, en la tecnología informática y de telecomunicaciones, etc. El uso y demanda del cobre es un buen indicador del nivel de crecimiento y desarrollo de los países y las estadísticas indican que en épocas de crisis económicas su consumo decrece ${ }^{2,3}$.

Otra aplicación ampliamente conocida, es su uso en las cañerías que transportan el agua de consumo para preservar su calidad sanitaria. Desde la antigüedad eran conocidas en forma empírica estas propiedades higiénicas que hoy sabemos responden a las propiedades antimicrobianas del cobre. Civilizaciones como los griegos, romanos, egipcios, aztecas y mayas, utilizaban el cobre en la fabricación de vasijas para preservar agua y alimentos, como también en la fabricación de instrumentos quirúrgicos y en la curación de heridas. En el siglo XVII se descubrió la capacidad del cobre para combatir plagas en la agricultura, específicamente para controlar infecciones por hongos en plantaciones de trigo. Hoy en día están disponibles en el mercado diferentes pesticidas que contienen cobre en diversas formulaciones químicas, los cuales se utilizan en agricultura. También existen pinturas con cobre usadas en embarcaciones para evitar adherencia de microorganismos y, secundariamente, crustáceos.

\section{Evidencias de la capacidad antimicrobiana del cobre}

\section{Actividad antibacteriana del cobre}

A pesar de contar con experiencias empíricas, solamente en los últimos años se han realizado investigaciones científicas que han permitido documentar las propiedades antimicrobianas del cobre y fomentar su uso en la práctica médica.

Diversos investigadores han demostrado, utilizando los criterios exigidos por la EPA (Environmental Protection Agency), que superficies de cobre o sus aleaciones son capaces de eliminar en horas 99,9\% de bacterias patógenas, entre ellas Staphylococcus aureus resistente a meticilina
(SAMR), Escherichia coli O157:H7, Pseudomonas aeruginosa, Enterobacter aerogenes, Listeria monocytogenes, Salmonella entérica, Campylobacter jejuni, Legionella pneumophila, Clostridium difficile y Mycobacterium tuberculosis ${ }^{4-8}$.

Resultados consistentes, bajo las condiciones establecidas por la $E P A$, demuestran la efectividad del cobre en la eliminación de bacterias patógenas a temperatura ambiente, a diferencia del acero inoxidable. En estos estudios el cobre eliminó de manera rápida el SAMR en $90 \mathrm{~min}$, mientras que con el acero inoxidable no se observó disminución en la concentración bacteriana después de $6 \mathrm{~h}$ (360 $\mathrm{min}$ ). En la aleación de bronce, que contiene $80 \%$ de cobre, el SAMR fue completamente eliminado en $270 \mathrm{~min}$.

Otro estudio, con cepas de SAMR, evaluó el efecto antimicrobiano del cobre en las superficies comunes del ambiente hospitalario comparándolo con el acero inoxidable y algunos polímeros como el $A g I O N^{\circledR}$ o Microban $^{\circledR}$, en donde la efectividad del cobre fue validada al demostrar una reducción en las Unidades Formadoras de Colonias (UFC) de siete órdenes de magnitud en $75 \mathrm{~min}$, mientras que se observó una pequeña reducción en las otras superficies después de 6 h. Una revisión exhaustiva de la literatura mostró que otros materiales utilizados, exceptuando el cobre, no presentan de manera confiable esta propiedad antimicrobiana en superficies disponibles comercialmente $e^{4,5}$.

Experimentos con cepas de Pseudomonas aeruginosa han mostrado un efecto sinérgico entre cationes de cobre, $\mathrm{Cu}_{2+}$ y desinfectantes de amonio cuaternario, para ejercer acción bactericida sobre este patógeno que tiene especial habilidad para sobrevivir en ambientes con baja concentración de nutrientes y un mínimo de humedad, por lo cual coloniza con frecuencia a catéteres y diversos equipos médicos?.

Evidencias de laboratorio documentan la eficacia del cobre para eliminar esporas y formas vegetativas de Clostridium difficile, patógeno hospitalario asociado a brotes de IIH con elevada mortalidad. Estos estudios mostraron eliminación de esporas después de 24 h de exposición a cobre metálico y otro estudio muestra que este efecto ocurre a partir de los $30 \mathrm{~min}$ para las formas vegetativas y a las $3 \mathrm{~h}$ para las esporas, aún en presencia de materia orgánica ${ }^{10,11}$.

Es importante destacar que el efecto bactericida de las superficies de cobre se relaciona directamen- 
te con la concentración, siendo el efecto máximo para el cobre metálico $(99,9 \%)$ y se mantiene en aleaciones que contengan al menos $70 \%$ de cobre.

Con el respaldo de la evidencia científica acumulada, el 25 de marzo de 2008, la EPA registró al cobre como el primer y único metal con propiedades antibacterianas, autorizando la difusión de conceptos importantes, entre ellos que "las superficies de cobre eliminan 99,9\% de los patógenos bacterianos después de $2 \mathrm{~h}$ de exposición" y certificando que superficies de cobre metálico y sus aleaciones son antimicrobianos naturales, poseen eficacia antimicrobiana de larga duración, tienen un efecto autodesinfectante y son superiores a otros revestimientos disponibles en el mercado. Este registro autoriza el uso de superficies de cobre en ambientes hospitalarios.

Adicionalmente, estudios de laboratorio realizados por nuestro grupo han demostrado que superficies de cobre, además de matar bacterias por contacto directo, impiden la adherencia e inhiben la multiplicación de cepas clínicas de los principales agentes asociados a infecciones intrahospitalarias, entre ellos SAMR, Klebsiella pneumoniae multiresistente, y Acinetobacter baumanii multiresistente, a diferencia de lo observado en superficies de acero inoxidable, en las cuales las bacterias se adhieren en forma rápida y muy eficiente (Prado V, enviado a publicar).

\section{Mecanismos involucrados en la actividad bactericida del cobre}

El mecanismo íntimo que explica la actividad antibacteriana del cobre no está totalmente dilucidado. Un elemento crucial en la actividad antibacteriana es la capacidad del cobre para ceder y aceptar electrones en un proceso continuo. Algunos estudios sugieren que el cobre, en concentraciones elevadas, tiene un efecto tóxico sobre las bacterias debido a la liberación de radicales de hidroperóxido, los iones de cobre potencialmente podrían sustituir iones esenciales para el metabolismo bacteriano como el hierro, interfiriendo inicialmente con la función de la membrana celular y luego a nivel del citoplasma alterando la síntesis proteica, ya sea inhibiendo la formación de proteínas o provocando la síntesis de proteínas disfuncionales, alterando la actividad de enzimas esenciales para el metabolismo bacteriano ${ }^{12,13}$.

En la Tabla 1 se describe una secuencia de eventos en la actividad bactericida.
Otros estudios sugieren que el cobre puede ejercer un efecto bacteriostático o bactericida, dependiendo de su concentración en el medio ${ }^{12}$. De acuerdo a nuestras observaciones en cepas clínicas productoras de biopelículas, el cobre es capaz, además, de inhibir la formación de estas comunidades multicelulares que se rodean de polímeros extracelulares, que facilitan la adherencia y colonización de superficies (Prado V, enviado a publicar).

\section{Actividad antiviral del cobre}

El cobre también ha demostrado capacidad para destruir virus de gran importancia médica, entre ellos virus influenza A y virus de inmunodeficiencia humana, $\mathrm{VIH}$, en concentraciones tan bajas como 0,16 a 1,6 mM. La elaboración de filtros con óxido de cobre ha permitido eliminar en forma eficiente el riesgo de transmisión de VIH a través de fluidos ${ }^{14}$. Los mecanismos involucrados en la actividad antiviral son la inactivación de una enzima proteasa importante para la replicación viral y daño a nivel de la envoltura fosfolipídica ${ }^{14-16}$.

\section{Actividad antifúngica del cobre}

Diferentes especies de hongos, entre ellas Candida albicans, patógeno importante en pacientes inmunodeprimidos, son inhibidas en su crecimiento y luego destruidas, en contacto con superficies de cobre. Estudios recientes indican que la actividad antifúngica ocurre mediante un proceso complejo denominado "muerte por contacto" en el cual se produce fundamentalmente un daño a la membrana citoplasmática, que se depolariza; no está claro si el daño afecta a las proteínas o los lípidos de la membrana. Esto facilita la entrada de iones de cobre a la célula, amplificando el daño y

\section{Tabla 1. Secuencia propuesta para eliminación de bacterias en superficies de cobre}

1. Iones de cobre se desprenden de superficies de cobre, penetran a la célula bacteriana y causan daño a la membrana citoplasmática

2. Ruptura de la membrana citoplasmática favorece entrada de iones cobre, esto conduce a disfunción de la membrana y aumento del estrés oxidativo

3. A nivel de citoplasma se produce alteración de la síntesis proteica y daño funcional de enzimas esenciales

4. Muerte celular y degradación del ADN bacteriano 
secundariamente se produce un aumento en el estrés oxidativo, sin apreciar daño aparente del ADN de estas células ${ }^{17}$.

\section{Importancia de las infecciones intrahospitalarias (IIH)}

Las infecciones intrahospitalarias (IIH) representan un problema serio a nivel mundial, ya que tienen graves consecuencias para los pacientes, tanto en morbilidad como letalidad. El riesgo de adquirir una IIH se estima en $4,5 \%$ de todas las hospitalizaciones y pueden tener diferente severidad, desde infección de una herida operatoria, infección del tracto urinario, septicemia, a neumonía. La mortalidad puede alcanzar hasta 27\% en pacientes de Unidades de Cuidados Intensivos $(\mathrm{UCI})^{18}$. Los factores de riesgo se relacionan con los procedimientos complejos hoy en día utilizados para manejar pacientes críticos y la mayor sobrevida de pacientes con enfermedades graves (Tabla 2).

El Centro de Control y Prevención de Enfermedades de Estados Unidos de Norteamérica (Centers for Disease Control and Prevention, CDC) estima que cada año se producen más de dos millones de infecciones intrahospitalarias en los Estados Unidos de Norteamérica, ocasionando alrededor de 90 mil muertes, con un costo de US $\$ 4,5$ a 5,0 mil millones por año ${ }^{18}$. Por otra parte, un estudio independiente realizado por un periódico en el año 2002 evaluó datos de 5.810 hospitales registrados en los Estados Unidos de Norteamérica, encontrando que la mortalidad por esta causa era de $103.000,14 \%$ superior que la informada por el CDC ${ }^{18,19}$.

Tabla 2. Factores de riesgo para adquirir infecciones intrahospitalarias

Enfermedades de base, no controladas

Inmunosupresión

Procedimientos invasivos

Presencia de cuerpos extraños

Hospitalización en UCI

Estadía prolongada en el hospital

Colonización por patógenos
Un aspecto adicional que dificulta el tratamiento de las IIH es el hecho que la mayoría de ellas son causadas por bacterias resistentes a múltiples antimicrobianos (multiresistentes) tales como el Staphylococcus aureus meticilino resistente (SAMR), Klebsiella pneumoniae productora de betalactamasa de espectro extendido, (BLEE), Acinetobacter spp multiresistente y el Enterococcus resistente a vancomicina (VRE). Dentro del arsenal terapéutico disponible, las opciones para el manejo de estas infecciones son limitadas y es preocupante observar que existe escasa actividad en investigación y desarrollo de nuevas moléculas antimicrobianas capaces de eludir los mecanismos de resistencia de estos patógenos. Es necesario contar con nuevas estrategias.

\section{Situación de las IIH en Chile}

Chile cuenta con un programa de control de infecciones bien establecido y la tasa de infecciones asociadas a la atención de salud es uno de los parámetros importantes para establecer la acreditación de los hospitales. Sin embargo, es un problema vigente que afecta en forma importante los costos en salud. Un estudio realizado en 2003 en hospitales públicos estimó en 70.000 los casos anuales de infecciones intrahospitalarias, las cuales prolongaban la estadía de los pacientes en un promedio de 10 días y aumentaba el uso de antibióticos en los pacientes afectados en dos a cuatro veces, con el consiguiente aumento en los costos de atención ${ }^{21}$.

Las tasas de incidencia son variables y las últimas cifras del Ministerio de Salud (MINSAL) indican que las tasas de neumonía en pacientes con ventilación mecánica fluctúan entre 4 y 19 por 1000 días de ventilación mecánica, las infecciones del tracto urinario de origen hospitalario tienen tasas entre 5 y 6 por 1.000 días de catéter urinario permanente y las bacteremias fluctúan entre $2,4 \mathrm{y}$ 3,6 por 1.000 días de exposición a procedimientos invasivos. http://www.redsalud.gov.cl/archivos/ infeccionesintrahospitalarias2007.pdf

\section{¿Cuál es la dinámica de la transmisión de patógenos dentro del hospital?}

El primer evento es el surgimiento de un microorganismo con ventajas biológicas, el cual 
se selecciona por su capacidad de sobrevivir en un ambiente adverso en el cual la presión de los antibióticos es importante, por lo que los patógenos nosocomiales presentan frecuentemente multiresistencia. Otro factor que ayuda a la supervivencia es la capacidad de producir biopelículas que les permiten adherirse a diferentes superficies y protegerse de amenazas externas. Hay evidencias que muestran que $S A M R$ puede permanecer por períodos de 7 días a 7 meses en superficies, $A c i$ netobacter spp entre 3 días a 5 meses, Enterococcus spp de 5 días a 4 meses y Clostridium difficile hasta por 5 meses $^{22}$. Por su parte, los virus, aunque menos resistentes a condiciones ambientales, pueden persistir hasta 2 días en el caso del virus influenza, 7 días el norovirus y 2 meses el rotavirus ${ }^{22}$.

Una vez adaptados al ambiente hospitalario ocurre el segundo paso, que es la interacción con los pacientes, de la cual puede resultar una colonización o una infección. En los pacientes estos patógenos se multiplican y se eliminan al ambiente contaminando superficies, telas o equipos médicos. A partir de estos reservorios los microrganismos pueden transmitirse de paciente a paciente, del personal sanitario o visitantes a los pacientes, principalmente mediante las manos o equipos médicos ${ }^{23,24}$.

Estos fenómenos explicarían la aparición esporádica de infecciones intrahospitalarias o de brotes.

El CDC ha publicado una guía para el control de las infecciones nosocomiales y ha recomendado el lavado de manos como la primera línea defensiva para minimizar la contaminación cruzada y las infecciones. Del mismo modo, recomienda regular la desinfección de equipos médicos que poseen superficies de contacto, especialmente aquellos en contacto directo con los pacientes. Sin embargo, todos los esfuerzos han resultado insuficientes ${ }^{18,25,26}$. A diferencia de estas prácticas de higiene intensivas, las superficies de cobre tienen un efecto auto-desinfectante que se mantiene en el tiempo.

Las superficies de contacto en las instalaciones del ambiente hospitalario, como manillas de puertas, placas de metal para empujar las puertas, barandas de camas, llaves del agua, porta suero y otros, han sido identificadas como reservorios y fuente de diseminación de agentes patógenos ${ }^{24,27}$.

Aunque los elementos de limpieza y desinfectantes recomendados pueden eliminar estos microorganismos, frecuentemente debido a las fisuras y rugosidad de las superficies, los productos de limpieza no logran penetrar al sitio donde se refugian estos organismos. Los materiales usados frecuentemente en infraestructura hospitalaria, como el acero inoxidable, no tienen la propiedad intrínseca del cobre para eliminar la contaminación microbiana.

\section{Superficies de cobre en el ambiente hospitalario}

Demostrada en el laboratorio la capacidad bactericida del cobre, el próximo paso fue evaluar si esta característica se mantenía en la vida real de ambientes clínicos. Los resultados de estudios clínicos son muy alentadores.

En un hospital de Birmingham, Inglaterra, se desarrolló un estudio durante 10 semanas, en el cual se intervinieron superficies en baños (asientos y tapas de WC) y manillas de puertas, que fueron reemplazadas por aleaciones de cobre (latón, aleación de cobre y zinc, 60-70\%). En este servicio clínico se mantuvieron objetos con superficies de plástico, aluminio o cromo. A las 5 semanas se intercambiaron los objetos. Se realizaron cultivos de las superficies y los resultados mostraron que la mediana de los recuentos de bacterias en las superficies de aleaciones de cobre fueron $90-100 \%$ menores que los recuentos de las superficies controles y patógenos como Staphylococcus aureus sensible a meticilina, Enterococcus resistente a vancomicina y Escherichia coli, fueron aislados solamente desde las superficies controles. Es interesante que el estudio microbiológico en este ensayo se realizó después de 6 meses de instaladas las superficies de cobre, mostrando que esta capacidad bactericida no se pierde en el tiempo ${ }^{28}$. Otro estudio más reciente de este grupo, en que se reemplazaron 14 objetos dentro del hospital y se realizaron recuentos bacterianos durante 24 semanas, muestra resultados similares, con reducción significativa de la carga bacteriana en 8 de los 14 objetos analizados ${ }^{29}$.

En Sudáfrica se realizó un estudio en un centro de atención primaria rural. Se escogieron superficies de alto contacto, como escritorios, armarios y alféizares de las ventanas. Se tomaron muestras durante 6 meses y se observó una reducción global de $71 \%$ de la carga bacteriana en las superficies con cobre ${ }^{30}$. 
En un hospital de Hamburgo en Alemania, se realizaron estudios similares en servicios de Oncología y Geriatría. En este ensayo se reemplazaron manillas de puertas e interruptores en salas de pacientes, salas de espera de visitas, y sitios de descanso del personal, manteniéndose el sistema de limpieza de rutina. Se realizaron cultivos diarios de las superficies durante 32 semanas, al cabo de este tiempo los recuentos de bacterias aerobias en las superficies con cobre se redujeron en 63\% promedio con relación a los controles, aluminio en las manillas y plástico en los interruptores. La reducción fue significativa en las manillas, los objetos con mayor carga bacteriana ${ }^{31}$.

Nuestro grupo participó en un estudio multi- céntrico, realizado en 2009-2010 en un hospital de Nueva York, dos hospitales de Charleston, en Carolina del Sur y el Hospital del Cobre de Calama. En el hospital de Calama se intervino en las Salas UCI, reemplazando superficies de alto contacto como barandas y manillas de camas, mesa del paciente, portasuero y apoya brazo de la silla de visitantes, por superficies de cobre metálico $(99,9 \%)$ o aleaciones. Se realizaron cultivos de bacterias aerobias durante 30 semanas, observando reducciones significativas en los recuentos promedios en todos los objetos de cobre (reducciones entre $49 \mathrm{y}$ 92\%) (V. Prado et al. $14^{\text {th }}$ International Congress on Infectious Diseases, Miami, Florida, USA, 9-12 Marzo, 2010) (Figura 1, Figura 2).
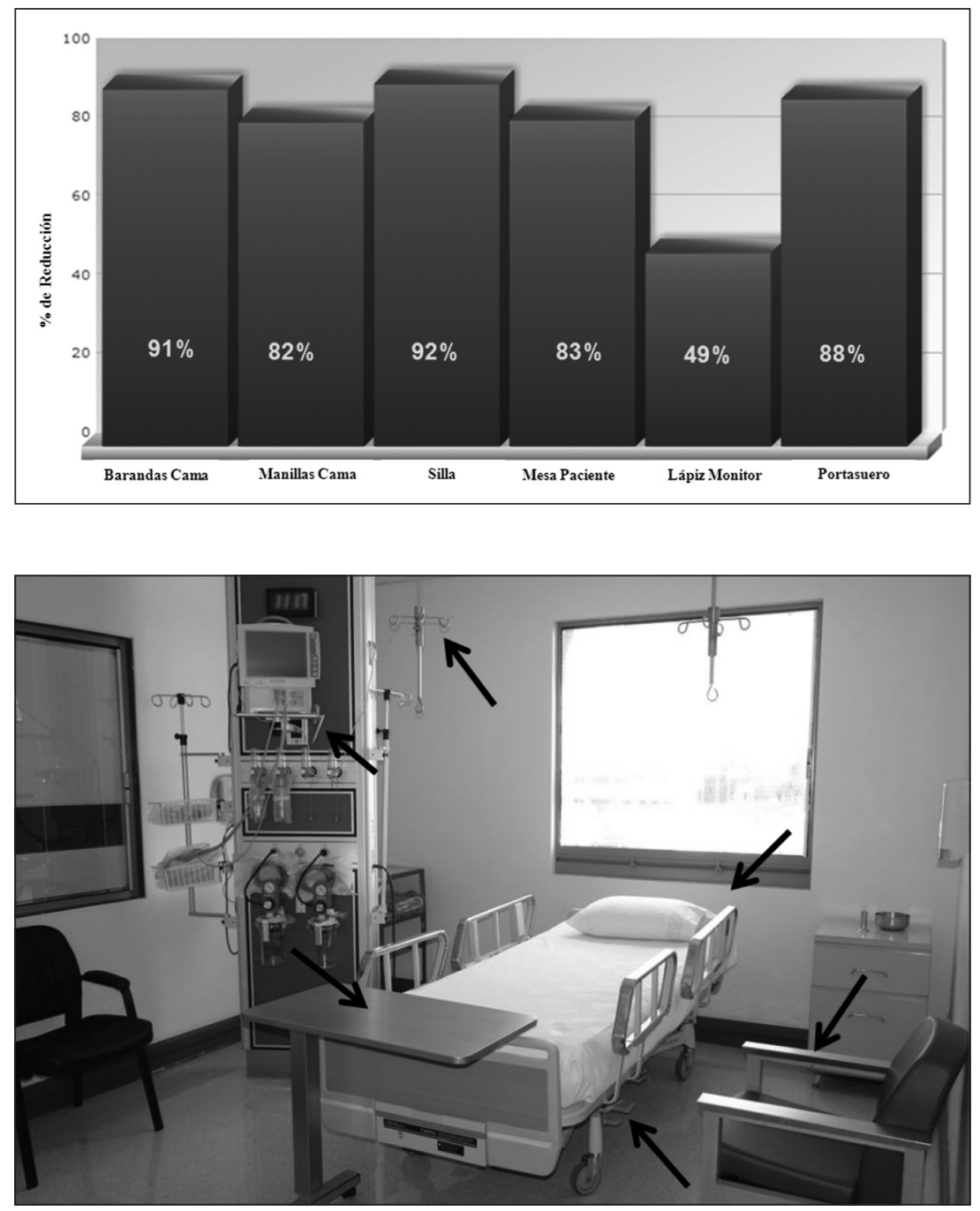

Figura 1. Porcentaje de reducción del recuento bacteriano total promedio por cada superficie de contacto en salas $\mathrm{UCl}$ con cobre, durante 30 semanas en el Hospital del Cobre de Calama. Reducción de bacterias viables (promedio de recuento de bacterias aerobias durante 30 semanas) en 6 superficies cubiertas con cobre o aleaciones en salas UCl del Hospital del Cobre de Calama. La mayor reducción se observó en barandas de camas (91\%) y apoya brazos de sillas de visitantes (92\%).

Figura 2. Imagen de una Sala de la Unidad de Cuidados Intensivos del Hospital del Cobre de Calama, en la cual se intervinieron 6 tipos de superficies con cobre: barandas y manillas de la cama, portasuero, mesa del paciente, apoya brazo de la silla de visitantes y lápiz del monitor. 


\section{Conclusión}

El cobre un elemento esencial para la vida humana, ha acompañado a la humanidad a través de los tiempos participando en diferentes áreas de su desarrollo, ayudando a mejorar la atención médica y la calidad de vida. Un concepto que se agrega es el reconocimiento de las propiedades antimicrobianas.

La aplicación del cobre o aleaciones, en superficies de contacto en el ambiente hospitalario ha demostrado ser eficiente para reducir la carga bacteriana ambiental, cualidad que se mantiene en el tiempo, lo cual significa menor riesgo de transmisión de patógenos a los pacientes y se debería considerar un complemento interesante junto a otras medidas de control de infecciones.

\section{Referencias}

1. Grass G, Rensing C, Solioz M. Metallic copper as an antimicrobial surface. Appl Environ Microbiol 2011; 77: 1541-7.

2. Lagos G, Henríquez H. Tendencias y desafíos que influencian el uso del cobre.2004. www.procobre.org.

3. Lagos G, Henríquez H. Escenarios para la demanda y oferta de cobre en el siglo XXI. 2000. www.editec.c

4. Noyce JO, Michels H, Keevil CW. Potential use of copper surfaces to reduce survival of epidemic meticillinresistant Staphylococcus aureus in the healthcare environment. J Hosp Infect 2006; 63: 289-97.

5. Noyce JO, Michels H, Keevil CW. Use of copper cast alloys to control Escherichia coli O157 cross-contamination during food processing. Appl Environ Microbiol 2006; 72: 4239-44.

6. Mehtar S, Wiid I, Todorov SD. The antimicrobial activity of copper and copper alloys against nosocomial pathogens and Mycobacterium tuberculosis isolated from healthcare facilities in the Western Cape: an in vitro study. J Hosp Infect 2008; 68: 45-51.

7. Wilks SA, Michels H, Keevil CW. Survival of Escherichia coli $\mathrm{O} 157$ on a range of metal surfaces. Int J Food Microbiol 2005; 105: 445-54.

8. Faúndez G, Troncoso M, Navarrete P, Figueroa G. Antimicrobial activity of copper surfaces against suspensions of Salmonella enterica and Campylobacter jejuni. BMC Microbiol 2004; 4: 19-23.

9. Harrison JJ, Turner RJ, Joo DA, Stan MA, Chan CS, Allan $\mathrm{ND}$, et al. Copper and quaternary ammonium cations exert synergistic bactericidal and antibiofilm activity against Pseudomonas aeruginosa. Antimicrob Agents Chemother 2008; 52: 2870-81.

10. Weaver L, Michels HT,Keevil CW. Survival of Clostridium difficile on copper and steel: future options for hospital higiene. J Hosp Inf 2008; 68: 145-51.

11. Wheeldon LJ, Worthington T, Lambert PA, Hilton AC, Lowden CJ, Elliot TSJ. Antimicrobial efficacy of copper surfaces against spores and vegetative cells of Clostridium difficile: the germination theory. J Antimicrobial Chem 2008; 52: 522-5.

12. Gordon AS, Howell LD, Harwood V. Responses of diverse heterotrophic bacteria to elevated copper concentrations. Can J Microbiol 1994; 40: 408-11.

13. Rodríguez-Montelongo L, De la Cruz -Rodríguez L, Farías RN, Mass EM. Membrane-associated redox cycling of copper mediates hydroproxide toxicity in Escherichia coli. Biochim Biophys Acta.1993; 1144: 77-84.

14. Borkow G, Lara HH, Covington CY, Nyamanti A, Gabbay J. Deactivation of human immunodeficiency virus type 1 in medium by copper oxide-containing filters. Antimicrob Agents Chemother 2008; 52: 518-25.

15. Sagripanti JL and Lighfoote MM. Cupric and ferric ions inactivate HIV. AIDS. Res Hum Retrov 1996; 12: 333-7.

16. Karlstrom AR, and Levine R. Cppers inhibits the poteasa from human immunodeficiency virus 1 by both cysteine-dependent and cysteine-independent mechanisms. Proc Natl Acad Sci USA. 1991; 88: 5552-6.

17. Quaranta D, Krans T, Espirito Santo C, Elowsky C G, Domaille DW, Chang CJ, and Grass G. Mechanisms of contact-mediated killing of yeast cells on dry metallic copper surfaces. Appl Environ Microbiol 2011; 77: 41626.

18. Gaynes R, Richards C, Edwards J, Emori TG, Peavy G, J. Tolson, and NN ISN Hospitals. Feeding Back Surveillance Data to Prevent Hospital-Acquired Infections. Emerg Infect Diseases 2001; 7: 295-8.

19. Berens MJ. Investigation: unhealthy hospitals: Drugresistant germs adapt, thrive beyond hospital walls. Chicago Tribune, 2002, pag. 1-5, Online Edition ed, Chicago.

20. Berens MJ. Investigation: unhealthy hospitals: Infection epidemic carves deadly path. Chicago Tribune, 2002, pag. 1-6, Online Edition ed, Chicago.

21. Brenner P, Nercelles P, Pohlenza M, Otaíza F. Costo de las infecciones intrahospitalarias en hospitales chilenos de alta y mediana complejidad. Rev Chil Infect 2003; 20 : 285-90.

22. Kramer A, Schwebke I, Kampf G. How long do nosocomial pathogens persist on inanimate surfaces? A systematic review. BMC Infectious Diseases 2006; 6: 130-41.

23. Bhalla A, Pultz NJ, Gries DM, Ray AJ, Eckstein EC, Aron 
DC et al. Acquisition of nosocomial pathogens on hands after contact with environmental surfaces near hospitalized patients. Infect Control Hosp Epidemiol 2004; 25: 164-7.

24. Blythe D, Keenlyside D, Dawson SJ, Galloway A. Environmental contamination due to methicillin-resistant Staphylococcus aureus (MRSA). J Hosp Infect 1998; 38: 67-9.

25. Fillaux J, Dubouix A, Conil JM, Laguerre J, Marty N. Retrospective Analysis of Multidrug-Resistant Acinetobacter baumannii Strains Isolated During a 4- Year Period in a University Hospital. Infect Control Hosp Epidemiol 2006; 27: 647-53.

26. Wilks M, Wilson A, Warwick S, Price E, Kennedy D, Ely A, et al. Control of an Outbreak of Multidrug-Resistant Acinetobacter baumannii calcoaceticus Colonization and Infection in an Intensive Care Unit (ICU) Without Closing the ICU or Placing Patients in Isolation. Infect Control Hosp Epidemiol 2006; 27: 654-8.
27. Neely AN. A survey of gram-negative bacteria survival on hospital fabrics and plastics. J Burn Care Rehabil 2000; 21: 523-7.

28. Casey AL, Adams D, Karpanen TJ, Lambert PA, Cookson $\mathrm{BD}$, Nightingale $\mathrm{P}$, et al. Role of copper in reducing hospital environment contamination. J Hosp Infect 2010; 74: 72-7.

29. Karpanen TJ, Casey AL, Lambert PA, Cookson BD, Nightingale P, Miuszenko L, et al. The antimicrobial efficacy of copper alloy furnishing in the clinical environment: a crossover study. Inf Control Hosp Epidemiol 2012; 33: 3-9.

30. Marais F, Methar S, Chalkley L. Antimicrobial efficay of copper touch surfaces in reducing environmental bioburden in a South African community healthcare facility. J Hosp Inf. 2010; 74: 80-2.

31. Mikolay A, Huggett S, Tikana L, Braun J, Nies DH. Survival of bacteria on metallic copper surfaces in ahospital trial. Appl Microbiol Biotechnol 2010: 87: 1875-9. 